Results Satisfaction that women have during labour and delivery is beyond their expectations. As follows from the hospital, expectations and satisfaction of women to improve. However, the expectation of women was not a predictor of perception, as opposed to satisfaction.

Conclusion Creating a space for reflection among professionals and managers of health institutions, indicating paths to follow and encouraging behavioural changes.

\section{PL.96 AN AUDIT ON MAJOR POSTPARTUM HAEMORRHAGE AT QUEENS HOSPITAL, ROMFORD}

doi:10.1136/archdischild-2013-303966.278

O Turan, M Hui. Queen's Hospital, London, UK

Introduction Haemorrhage is a leading cause of maternal death and morbidity. Major postpartum haemorrhage (PPH) is loss of more than 1 Litre of blood from the genital tract postnatally.

Objectives To undertake a retrospective audit of patients who underwent a major PPH. Identify areas of good practise and make recommendations to improve standard of care.

Materials and Methods 197 cases were identified from the electronic database (September 2011 to March 2012) and 127 notes reviewed retrospectively. Data was analysed for patient demographics, risk factors, delivery and documentation. Reference was made to the RCOG Green-Top Guideline No. 52 and a previous local audit.

Results The majority were Caucasian (40\%), nulliparous (50\%), aged 18-30 (61\%) with a normal BMI (47\%). Most were delivered by Caesarean section (69\%) at term (61\%). $60 \%$ of babies weighed between 3 and $4 \mathrm{~kg}$ and $10 \%$ of women were estimated to have lost $2.5 \mathrm{~L}$ or more. 27 patients required blood transfusion of up to 10 units. There were 6 manual removals of placentae and 8 other surgical interventions, including one hysterectomy.

Discussion There was no correlation between the number of risk factors and total blood loss. An improvement in the completion $\mathrm{PPH}$ proformas and patient debriefing was found. However, there appeared to be deterioration in emergency call-out (2222) and incident reporting in comparison to previous audit data.

Recommendations We recommend refresher training and emergency drills for identification and management of the critically ill patient. Improve awareness of local and regional guidelines and re-audit in one year.

\section{PL.97 PROSPECTIVE AUDIT FOR INDUCTION OF LABOUR WITH PROPESS}

doi:10.1136/archdischild-2013-303966.279

SN Malik, H Kansara, HA Mousa. University Hospitals of Leicester, Leicester, LE1 5WW, UK

\footnotetext{
Aim to assess the success rate of IOL using propess.

Method A prospective audit of IOL was carried out in a large teaching hospital. Special data sheet was used for collection of data. We have included women with a singleton viable pregnancy in a cephalic presentation and who were admitted for IOL at $\geq 37$ weeks gestation. We have excluded cases with history of rupture of membranes.

Results Despite of 24 hours of propess use, 31/100 (31\%) additionally needed Prostin as adjuvant method for IOL. 54/100 (54\%) needed syntocinon for augmentation of labour. Hyperstimulation syndrome with CTG changes were observed in 7/100 (7\%) of cases (five after propess and two after oxytocin infusion). Vaginal delivery was achieved in 73/100 (73\%). It was observed that 73/100 (73\%) of women delivered between 48 hours of IOL. Primary postpartum haemorrhage was noted in 15/100 (15\%). There was no neonatal admission.
}

Conclusion IOL with proess is successful method with about $73 \%$ success rate. However, clinicians should be aware that up to $30 \%$ of cases will need extra prostin. Cost analysis is required to evaluate methods that could be used to reduce cost and duration of admission.

\section{PL.98 INDICATIONS AND OUTCOMES IN CAESAREAN SECTION - A PROSPECTIVE AUDIT}

doi:10.1136/archdischild-2013-303966.280

OJ Barney, T Mousa, T Lessells, O Navti. University Hospitals Leicester, Leicester, UK

The PCT required an audit to be completed across the University Hospitals Leicester Trust in the light of new NICE guidance on Caesarean section for maternal request. This was combined with an audit of infection control and thromboprophylaxis measures for CQUIN targets. An audit of Vaginal Birth After Caesarean as compared with projected outcome of VBACs published by the RCOG was opportunistically carried out at the same time.

Data were prospectively collected on patients undergoing CS in January 2012 by the theatre team in 171/191 (89\%) of all patients undergoing caesarean section and VBAC data were collected from the patients' computerised and paper notes.

Targets were met for Antibiotic prophylaxis and use of clippers, VTE prophylaxis, Diabetic control and counselling against CS for maternal request.

Targets were not met for written and verbal debriefing post CS, nor prescribing of Stellisept.

\section{Additional findings}

- Diabetes rate $6 \%$ across UHL

- Hypertension rate $16 \%$ across UHL

- $100 \%$ UHL patients had an AN discussion about mode of delivery

- CS Rate $21 \%$ across UHL

- Only $24 \%$ those that could have attempted VBAC chose ERCS (LRI)

- VBAC success rate $64.3 \%$ in those who attempted it (LRI)

- CS Maternal request - 4 cases/171 2.3\%

- NICE CG 132 1.2.9.2

- Documented adequate counselling $\quad 100 \%$

- Explored reasons for request 100\%

Conclusion The audit was reassuring in not only its prophylactic measures but also that very reasonable successful VBAC rates and CS rates are achievable in even a tertiary unit.

\section{PL.99 FETAL BLOOD SAMPLING DOCUMENTATION - REAUDIT}

doi:10.1136/archdischild-2013-303966.281

${ }^{1}$ A Yulia, ${ }^{1} \mathrm{~F}$ Mure, ${ }^{2} \mathrm{C}$ Pun, ${ }^{1} \mathrm{~J}$ Oghoetuoma. ${ }^{1}$ County Durham and Darlington NHS Foundation Trust, Darlington, UK; ${ }^{2}$ North Cumbria NHS Trust, Carlisle, UK

Introduction The intrapartum assessment of fetal well-being remains a challenge to obstetricians, especially given the on-going concern for increasing caesarean section rates and malpractice litigation. Several techniques may be used to evaluate the fetus during labour including direct analysis of fetal blood obtained from via scalp sampling. It is important that when fetal blood sampling (FBS) is performed, clear documentation should be done. At Darlington Memorial Hospital (DMH), a re-audit was performed to assess the documentation on FBS, according to the recommendations stated in the Trust Guidelines.

Aim To determine whether there are any improvements in documentation on FBS compared to previous audit performed in 2010

Methods A retrospective audit of the 22 cases was performed from $1^{\text {st }}$ July to $30^{\text {th }}$ September 2011 at DMH. Data were collected from the labour ward register, fetal blood sampling record and medical notes.

Results A total of 22 cases were included. Compared to 2010, there was improvement in the documentation of the cord blood being 